\title{
Application of Laplace Transforms Technique in Solving Second Order Complex Differential Equations
}

\author{
S. Usman ${ }^{1 *}$, I. Abdullahi ${ }^{1}$, B. G. Agaie ${ }^{1}$, N. A. Muhammad ${ }^{1}$,
} H. B. Yusuf ${ }^{2}$ and K. G. Ibrahim ${ }^{1}$

${ }^{1}$ Department of Mathematics, Federal University Dutse (FUD), PMB 7156, Jigawa State, Nigeria

${ }^{2}$ Department of Mathematics, Nigerian Army University Biu, PMB 1500, Borno State, Nigeria

Email: usman.sani@fud.edu.ng

\begin{abstract}
In this research work Complex Differential Equations of order two was studied and then solved using the Laplace transform technique. The Complex Differential Equations were separated into the real and imaginary parts and an expression for the solution form of the complex differential equation of order two with constant coefficient was obtained. The solution to the problems were then obtained using Laplace technique. The real and imaginary parts of the solution were further obtained using the inverse Laplace transform giving the results for the problem, and also illustrative examples are included to demonstrate the validity and applicability of the expression for the solution form of the problems.
\end{abstract}

Keywords: Laplace Transform Technique, Inverse Laplace Transform, Dirac Delta Function, Complex Differential Equation.

\section{INTRODUCTION}

In the field of mathematics, when it comes to the general solution to some type of equations especially the elliptic type of equation their solutions are not easily solved for. And so when considering such type of equations, the partial differential equation system with even number of independent variable can be transformed to a complex partial differential equation giving a clear chance of obtaining the solution to the equation using complex methods.

Yusufoglu (2006) studied the numerical solution of duffing equation by the Laplace Algorithm where the principle of Laplace Decomposition Algorithm (LDA) was described and also its advantages and drawbacks. Examples were also studied to show with numerical results how the LDA works efficiently. Kexue and Jigen (2011) gave sufficient condition in guaranteeing the rationality of solving constant coefficient fractional differential equation using Laplace transform method. Yin et al. (2013) developed a new scheme to obtain the 
exact solutions for the singular initial value problems (IVPs) of Lane-Emden type of problem where the new scheme was deduced from the Modified Laplace Decomposition Method (MIDM). The problems considered consist of both linear and nonlinear cases and it was seen that the exact solution exist in the zeroth component yielding the exact solution in two iterations applying the proposed method. Exact solution of some linear fractional differential equations by Laplace transform was studied by Saheed (2014) where Laplace transform was applied in solving linear fractional-order differential equation. It was observed that the Laplace transform is powerful and efficient for obtaining analytic solution of linear fractional differential equations. Mohamed and Torky (2013) solved for the approximate solutions for Whitham-Broer-Kamp shallow water model, the coupled nonlinear reaction equation alongside system of Hirota-Satsuma coupled KdV using the Laplace Decomposition Method (LDM) and Pade approximation. It was noted the scheme found the solutions without any discretization or restrictive assumption and also free from round off errors reducing the numerical computations to a great extent. Numerical study for systems of fractional differential equations via Laplace transform was considered by Gupta et al. (2015) where numerical algorithm for solving system of fractional differential equation was proposed using Homotopy analysis transform method. The numerical results show that the approach is easy to implement and accurate when applied to various fractional differential equations. Murat (2017) studied the application of Laplace transforms on complex differential equations of order one where the real and imaginary parts of the solutions were obtained using the inverse Laplace transform. Dinesh (2018) investigated the application of Laplace Transform for solving various differential equations with constant variable coefficient using linear ordinary differential equations to illustrate the new technique. Kazem (2013) applied Laplace transform in solving linear fractional order differential equation where the fractional differential equation was transform into algebraic equations and then solving them. The technique was used to obtain an exact solution of some linear fractional differential equations.

Based on the above mentioned literatures, investigation carried out are on first order complex partial differential equations, partial differential equations and nonlinear partial differential equation using Laplace transform method, but on the solution of second order complex partial differential equation with constant coefficient using Laplace transforms have not been studied which form the bases of this paper.

\section{METHODOLOGY}

\section{Basic Definitions and Theorems}

Definition 1. Let $F(t)$ be a function of $t>0$. Laplace transform of $F(t)$ is defined. $L(F(t))=f(s)=\int_{0}^{\infty} e^{-s t} f(t) d t$

Since the integral of equation (1) is a function of $s$, so we write $L(F(t))=f(s)$

Theorem 1. If $F^{(n)}(t)$ is continuous, then

$L\left(F^{(n)}(t)\right)=s^{n} f(s)-s^{n-1} F(0)-s^{n-2} F^{\prime}(0)-s^{n-3} F^{\prime \prime}(0)-\cdots-F^{n-1}(0)$

where $L(F(t))=f(s)$

Theorem 2. Laplace transforms of partial derivatives of $u(x, t)$ are given as follows.

$$
L\left[\frac{\partial u}{\partial t}\right]=s U(x, s)-u(x, 0)
$$

$$
L\left[\frac{\partial u}{\partial x}\right]=\frac{\partial U(x, s)}{\partial x}
$$


(iii) $\quad L\left[\frac{\partial^{2} u}{\partial t^{2}}\right]=s^{2} U(x, s)-s u(x, 0)-u^{\prime}(x, 0)$

(iv) $\quad L\left[\frac{\partial^{2} u}{\partial x^{2}}\right]=\frac{\partial^{2} U(x, s)}{\partial x^{2}}-\frac{\partial u(x, 0)}{\partial x}$

where $U(x, s)=L[u(x, t)]$.

\subsection{Complex Derivatives}

Let $w=w(z, \bar{z})$ be a complex function, and $z=x+y i, w(z, \bar{z})=u(x, y)+i v(x, y)$. First order derivatives according to $z$ and $\bar{z}$ of $w(z, \bar{z})$ are defined as

$$
\begin{gathered}
\frac{\partial w}{\partial z}=\frac{1}{2}\left(\frac{\partial w}{\partial x}-i \frac{\partial w}{\partial y}\right) \\
\frac{\partial w}{\partial \bar{z}}=\frac{1}{2}\left(\frac{\partial w}{\partial x}+i \frac{\partial w}{\partial y}\right)
\end{gathered}
$$

and for the second order are defined as

$\frac{\partial^{2} w}{\partial z^{2}}=\frac{1}{2}\left(\frac{\partial^{2} w}{\partial x^{2}}-i \frac{\partial^{2} w}{\partial y^{2}}\right)$

$\frac{\partial^{2} w}{\partial \bar{z}^{2}}=\frac{1}{2}\left(\frac{\partial^{2} w}{\partial x^{2}}+i \frac{\partial^{2} w}{\partial y^{2}}\right)$

2.3. Solutions of complex differential equations of second order with constant coefficients.

Theorem 3. Let $A, B, C$ be a real constants, $F(z, \bar{z})$ is a polynomial of $z, \bar{z}$ and $w=u+i v$ is a complex function. Then the solution of

$A \frac{\partial^{2} w}{\partial z^{2}}+B \frac{\partial^{2} w}{\partial \bar{z}^{2}}+C w=F(z, \bar{z})$

with the initial condition

$w(x, 0)=f(x)$

is given as

$u(x, y)$

$=L^{-1}\left[\frac{(A+B) \frac{\partial^{2}}{\partial x^{2}}\left(2 F_{1}^{*}+(A-B)\left(s v(x, 0)+v^{\prime}(x, 0)\right)\right)+2 C\left(2 F_{1}{ }^{*}+(A-B)\left(s v(x, 0)+v^{\prime}(x, 0)\right)\right)-s^{2}(A-B)\left(2 F_{2}{ }^{*}+(B-A)\left(s u(x, 0)+u^{\prime}(x, 0)\right)\right)}{\left((A+B) \frac{\partial^{2}}{\partial x^{2}}+2 C\right)^{2}+s^{2}(A-B)^{2}}\right]$

$v(x, y)$

$=L^{-1}\left[\frac{(A+B) \frac{\partial^{2}}{\partial x^{2}}\left(2 F_{2}{ }^{*}+(B-A)\left(s u(x, 0)+u^{\prime}(x, 0)\right)\right)+2 C\left(2 F_{2}{ }^{*}+(B-A)\left(s u(x, 0)+u^{\prime}(x, 0)\right)\right)-s^{2}(B-A)\left(2 F_{1}^{*}+(A-B)\left(s v(x, 0)+v^{\prime}(x, 0)\right)\right)}{\left((A+B) \frac{\partial^{2} u u}{\partial x^{2}}+2 C\right)^{2}+s^{2}(A-B)^{2}}\right]$

and hence the proof is

$A \frac{\partial^{2} w}{\partial z^{2}}+B \frac{\partial^{2} w}{\partial \bar{z}^{2}}+C w=F(z, \bar{z})$

Obtaining the following equality by substituting equations (9) and (10) in (15) yields $A \frac{1}{2}\left(\frac{\partial^{2} w}{\partial x^{2}}-i \frac{\partial^{2} w}{\partial y^{2}}\right)+B \frac{1}{2}\left(\frac{\partial^{2} w}{\partial x^{2}}+i \frac{\partial^{2} w}{\partial y^{2}}\right)+C w=F_{1}(x, y)+i F_{2}(x, y)$

If we write $w=u+i v$ in equation (16) we then have

$A\left(\frac{\partial^{2} u}{\partial x^{2}}+i \frac{\partial^{2} v}{\partial x^{2}}-i \frac{\partial^{2} u}{\partial y^{2}}+\frac{\partial^{2} v}{\partial y^{2}}\right)+B\left(\frac{\partial^{2} u}{\partial x^{2}}+i \frac{\partial^{2} v}{\partial x^{2}}+i \frac{\partial^{2} u}{\partial y^{2}}-\frac{\partial^{2} v}{\partial y^{2}}\right)+2 C(u+i v)=2 F_{1}(x, y)+$

$2 i F_{2}(x, y)$

Separating equation (17) into real and imaginary parts, then the following system of equations are obtained

$$
\begin{aligned}
& (A+B) \frac{\partial^{2} u}{\partial x^{2}}+(A-B) \frac{\partial^{2} v}{\partial y^{2}}+2 C u=2 F_{1}(x, y) \\
& (A+B) \frac{\partial^{2} v}{\partial x^{2}}+(B-A) \frac{\partial^{2} u}{\partial y^{2}}+2 C v=2 F_{2}(x, y)
\end{aligned}
$$


Using the Laplace transform on equations (18) and (19) above, we get the following equalities

$$
\begin{aligned}
& (A+B) \frac{\partial^{2} U}{\partial x^{2}}+(A-B)\left(s^{2} V-s v(x, 0)-v^{\prime}(x, 0)\right)+2 C U=2 F_{1}{ }^{*} \\
& (A+B) \frac{\partial^{2} V}{\partial x^{2}}+(B-A)\left(s^{2} U-s u(x, 0)-u^{\prime}(x, 0)\right)+2 C V=2 F_{2}{ }^{*}
\end{aligned}
$$

where $U, V, F_{1}{ }^{*}, F_{2}{ }^{*}$ are Laplace transforms of $u, v, F_{1}, F_{2}$ respectively. Rearranging equation (20) and (21) and then solving using Cramer's rule yields

$$
\begin{aligned}
& (A+B) \frac{\partial^{2} U}{\partial x^{2}}+2 C U+s^{2}(A-B) V=\left(s v(x, 0)+v^{\prime}(x, 0)\right)(A-B)+2 F_{1}{ }^{*} \\
& s^{2}(B-A) U+2 C U+(A+B) \frac{\partial^{2} V}{\partial x^{2}}+2 C V=\left(s u(x, 0)+u^{\prime}(x, 0)\right)(B-A)+2 F_{2}{ }^{*} \\
& \left|\begin{array}{cc}
(A+B) D+2 C & s^{2}(A-B) \\
s^{2}(B-A) & (A+B) D+2 C
\end{array}\right|=((A+B) D+2 C)^{2}+s^{4}(A-B)^{2}
\end{aligned}
$$

$U=\frac{\left|\begin{array}{cc}\left(s v(x, 0)+v^{\prime}(x, 0)\right)(A-B)+2 F_{1}{ }^{*} & s^{2}(A-B) \\ \left(s u(x, 0)+u^{\prime}(x, 0)\right)(B-A)+2 F_{2}{ }^{*} & (A+B) D+2 C\end{array}\right|}{((A+B) D+2 C)^{2}+s^{4}(A-B)^{2}}$

$U=$

$\frac{(A+B) \frac{\partial^{2}}{x^{2}}\left[2 F_{1}{ }^{*}+(A-B)\left(s v(x, 0)+v^{\prime}(x, 0)\right)\right]+2 C\left[2 F_{1}{ }^{*}+(A-B)\left(s v(x, 0)+v^{\prime}(x, 0)\right)\right]-s^{2}(A-B)\left[2 F_{2}{ }^{*}+(B-A)\left(s u(x, 0)+u^{\prime}(x, 0)\right)\right]}{((A+B) D+2 C)^{2}+s^{4}(A-B)^{2}}$

$$
\begin{aligned}
& V=\frac{\left|\begin{array}{cc}
(A+B) D+2 C & \left(s v(x, 0)+v^{\prime}(x, 0)\right)(A-B)+2 F_{2}{ }^{*} \\
s^{2}(B-A) & \left(s u(x, 0)+u^{\prime}(x, 0)\right)(B-A)+2 F_{1}{ }^{*}
\end{array}\right|}{((A+B) D+2 C)^{2}+s^{4}(A-B)^{2}} \\
& V=\frac{(A+B) \frac{\partial^{2}}{x^{2}}\left[2 F_{2}{ }^{*}+(B-A)\left(s u(x, 0)+u^{\prime}(x, 0)\right)\right]+2 C\left[2 F_{2}{ }^{*}+(B-A)\left(s u(x, 0)+u^{\prime}(x, 0)\right)\right]-s^{2}(B-A)\left[2 F_{1}{ }^{*}+(A-B)\left(s v(x, 0)+v^{\prime}(x, 0)\right)\right]}{((A+B) D+2 C)^{2}+s^{4}(A-B)^{2}}
\end{aligned}
$$

(26)

The following are obtained from the inverse Laplace transform of equations (24) and (26) $U(x, y)$

$$
\begin{aligned}
& =L^{-1}\left[\frac{(A+B) \frac{\partial^{2}}{x^{2}}\left[2 F_{1}{ }^{*}+(A-B)\left(s v(x, 0)+v^{\prime}(x, 0)\right)\right]+2 C\left[2 F_{1}{ }^{*}+(A-B)\left(s v(x, 0)+v^{\prime}(x, 0)\right)\right]-s^{2}(A-B)\left[2 F_{2}{ }^{*}+(B-A)\left(s u(x, 0)+u^{\prime}(x, 0)\right)\right]}{((A+B) D+2 C)^{2}+s^{4}(A-B)^{2}}\right] \\
& V(x, y) \\
& =L^{-1}\left[\frac{(A+B) \frac{\partial^{2}}{x^{2}}\left[2 F_{2}^{*}+(B-A)\left(s u(x, 0)+u^{\prime}(x, 0)\right)\right]+2 C\left[2 F_{2}^{*}+(B-A)\left(s u(x, 0)+u^{\prime}(x, 0)\right)\right]-s^{2}(B-A)\left[2 F_{1}^{*}+(A-B)\left(s v(x, 0)+v^{\prime}(x, 0)\right)\right]}{((A+B) D+2 C)^{2}+s^{4}(A-B)^{2}}\right]
\end{aligned}
$$

Equations (27) and (28) are the required solution form of the second order complex differential equation which on applying them to our examples we get the desired solution for them.

\section{RESULTS AND DISCUSSION}

Example 1. Consider the second order complex differential equation

$\frac{\partial^{2} w}{\partial z^{2}}+2 \frac{\partial^{2} w}{\partial \bar{z}^{2}}=3 z^{2}+2$

with the conditions

$w(x, 0)=x^{3}+x, w^{\prime}(x, 0)=3 x^{2}+1$ 
Applying the foresaid solution form to equation (29) subject to equation (30) where the coefficients of equation (29) are $A=1, B=2, C=0$ and $F(z, \bar{z})=3 x^{2}+2$. The Real and imaginary parts of $F(z, \bar{z})$ are $F_{1}(x, y)=3 x^{2}-3 y^{2}+2$ and $F_{2}(x, y)=6 x y$, and the Laplace transforms of $F_{1}(x, y)$ and $F_{2}(x, y)$ are

$F_{1}{ }^{*}(x, s)=L\left[F_{1}(x, y)\right]=\frac{3 x^{2}+2}{s}$ and $F_{2}{ }^{*}(x, s)=L\left[F_{2}(x, y)\right]=\frac{6 x}{s^{2}}$

applying the solution form yields

$$
\begin{aligned}
& u(x, y)=L^{-1}\left[\frac{3 \frac{\partial^{2}}{\partial x^{2}}\left(\frac{6 x^{2}+4}{s}-\frac{12}{s^{3}}\right)+s^{2}\left(\frac{12 x}{s^{2}}+s\left(x^{3}+x\right)+\left(3 x^{2}+1\right)\right)}{9 D^{2}+s^{4}}\right] \\
& u(x, y)=L^{-1}\left[\frac{\frac{36}{s}+12 x+s^{3}\left(x^{3}+x\right)+s^{2}\left(3 x^{2}+1\right)}{s^{4}\left(1+\frac{9 D^{2}}{s^{4}}\right)}\right] \\
& u(x, y)=L^{-1}\left[\frac{1}{s^{4}\left(1+\frac{9 D^{2}}{s^{4}}\right)}\left(\frac{36}{s}+12 x+s^{3}\left(x^{3}+x\right)+s^{2}\left(3 x^{2}+1\right)\right)\right] \\
& u(x, y)=L^{-1}\left[\frac{1}{s^{4}}\left(\frac{36}{s}+12 x+s^{3}\left(x^{3}+x\right)+s^{2}\left(3 x^{2}+1\right)-\frac{54 x}{s}-\frac{54}{s^{2}}\right)\right] \\
& u(x, y)=L^{-1}\left[\frac{36-54 x}{s^{5}}+\frac{12 x}{s^{4}}+\frac{x^{3}+x}{s}+\frac{3 x^{2}+1}{s^{2}}-\frac{54}{s^{6}}\right] \\
& u(x, y)=27 y^{5}+\frac{9 x y^{4}}{4}-\frac{3 y^{4}}{2}-2 x y^{3}-3 x^{2} y-x^{3}+x+y \\
& v(x, y)=L^{-1}\left[\frac{3 \frac{\partial^{2}}{\partial x^{2}}\left(\frac{12 x}{s^{2}}\right)-s^{2}\left(\frac{6 x^{2}+4}{s}-\frac{12}{s^{3}}-s\left(x^{3}+x\right)+\left(3 x^{2}+1\right)\right)}{9 D^{2}+s^{4}}\right] \\
& v(x, y)=L^{-1}\left[\frac{-s\left(6 x^{2}+4\right)+\frac{12}{s}+s^{3}\left(x^{3}+x\right)+s^{2}\left(3 x^{2}+1\right)}{9 D^{2}+s^{4}}\right] \\
& v(x, y)=L^{-1}\left[\frac{1}{s^{4}\left(1+\frac{9 D^{2}}{s^{4}}\right)}\left(-s\left(6 x^{2}+4\right)+\frac{12}{s}+s^{3}\left(x^{3}+x\right)+s^{2}\left(3 x^{2}+1\right)\right)\right] \\
& v(x, y)=L^{-1}\left[\frac{1}{s^{4}}\left(-s\left(6 x^{2}+4\right)+\frac{12}{s}+s^{3}\left(x^{3}+x\right)+s^{2}\left(3 x^{2}+1\right)+\frac{108}{s^{3}}-\frac{54 x}{s}-\frac{54}{s^{2}}\right)\right] \\
& v(x, y)=L^{-1}\left[\frac{-6 x^{2}-4}{s^{3}}+\frac{12}{s^{5}}+\frac{x^{3}+x}{s}+\frac{3 x^{2}+1}{s^{2}}+\frac{108}{s^{7}}-\frac{54 x}{s^{5}}-\frac{54}{s^{6}}\right] \\
& v(x, y)=x^{3}+3 x^{2} y-3 x^{2} y^{2}-\frac{9 x y^{4}}{4}+x+\frac{3 y^{6}}{40}-\frac{9 y^{5}}{20}+\frac{y^{4}}{2}-2 y^{2}+y
\end{aligned}
$$

hence the general solution form is

$w=u+i v=27 y^{5}+\frac{9 x y^{4}}{4}-\frac{3 y^{4}}{2}-2 x y^{3}-3 x^{2} y-x^{3}+x+y+i\left(x^{3}+3 x^{2} y-3 x^{2} y^{2}-\frac{9 x y^{4}}{4}+\right.$

$\left.x+\frac{3 y^{6}}{40}-\frac{9 y^{5}}{20}+\frac{y^{4}}{2}-2 y^{2}+y\right)$

Example 2. Solve the following problem

$2 \frac{\partial^{2} w}{\partial z^{2}}-\frac{\partial^{2} w}{\partial \bar{z}^{2}}=4 z+1$

with the conditions

$$
w(x, 0)=x^{2}+5 x, w^{\prime}(x, 0)=2 x+5
$$

Also applying the foresaid solution form to equation (34) subject to equation (35) where the coefficients of equation (33) are $A=2, B=-1, C=0$ and $F(z, \bar{z})=4 z+1$. The Real and imaginary parts of $F(z, \bar{z})$ are $F_{1}(x, y)=4 x+1$ and $F_{2}(x, y)=4 y$, and the Laplace transforms of $F_{1}(x, y)$ and $F_{2}(x, y)$ are

$$
F_{1}{ }^{*}(x, s)=L\left[F_{1}(x, y)\right]=\frac{4 x+1}{s} \text { and } F_{2}{ }^{*}(x, s)=L\left[F_{2}(x, y)\right]=\frac{4}{s^{2}}
$$

and applying the solution form yield

$$
u(x, y)=L^{-1}\left[\frac{\frac{\partial^{2}}{\partial x^{2}}\left(\frac{8 x+2}{s}\right)-3 s^{2}\left(\frac{8}{s^{2}}-3 s\left(x^{2}+5 x\right)+3(2 x+5)\right)}{D^{2}+9 s^{4}}\right]
$$




$$
\begin{aligned}
& u(x, y)=L^{-1}\left[\frac{-24+9 s^{3}\left(x^{2}+5 x\right)-9 s^{2}(2 x+5)}{D^{4}+9 s^{4}}\right] \\
& u(x, y)=L^{-1}\left[\frac{1}{9 s^{4}\left(1+\frac{D^{2}}{9 s^{4}}\right)}\left(-24+9 s^{3}\left(x^{2}+5 x\right)-9 s^{2}(2 x+5)\right)\right] \\
& u(x, y)=L^{-1}\left[\frac{1}{9 s^{4}}\left(1-\frac{D^{2}}{9 s^{2}}+\frac{D^{4}}{81 s^{8}}-\cdots\right)\left(-24+9 s^{3}\left(x^{2}+5 x\right)-9 s^{2}(2 x+5)\right)\right] \\
& u(x, y)=L^{-1}\left[\frac{1}{9 s^{4}}\left(-24+9 s^{3}\left(x^{2}+5 x\right)-9 s^{2}(2 x+5)-\frac{18}{s}\right)\right] \\
& u(x, y)=L^{-1}\left[-\frac{24}{s^{4}}+\frac{x^{2}+5 x}{s}-\frac{2 x+5}{s^{2}}-\frac{2}{s^{5}}\right] \\
& u(x, y)=-\frac{4 y^{3}}{9}+\left(x^{2}+5 x\right)-\frac{(2 x+5) y}{2}-\frac{y^{4}}{12}
\end{aligned}
$$

similarly

$$
\begin{aligned}
& v(x, y)=L^{-1}\left[\frac{\frac{\partial^{2}}{\partial x^{2}}\left(\frac{8}{s^{2}}\right)+3 s^{2}\left(\frac{8 x^{2}+2}{s}+3 s\left(x^{2}+5 x\right)+3(2 x+5)\right)}{D^{2}+9 s^{4}}\right] \\
& v(x, y)=L^{-1}\left[\frac{1}{9 s^{4}\left(1+\frac{D^{2}}{9 s^{4}}\right)}\left(3 s(8 x+2)+9 s^{3}\left(x^{2}+5 x\right)+9 s^{2}(2 x+5)\right)\right] \\
& v(x, y)=L^{-1}\left[\frac{1}{9 s^{4}}\left(1-\frac{D^{2}}{9 s^{2}}+\frac{D^{4}}{81 s^{8}}-\cdots\right)\left(3 s(8 x+2)+9 s^{3}\left(x^{2}+5 x\right)+9 s^{2}(2 x+5)\right)\right] \\
& v(x, y)=L^{-1}\left[\frac{1}{9 s^{4}}\left(3 s(8 x+2)+9 s^{3}\left(x^{2}+5 x\right)+9 s^{2}(2 x+5)-\frac{2}{s}\right)\right] \\
& v(x, y)=L^{-1}\left[\frac{8 x+2}{3 s^{3}}+\frac{x^{2}+5 x}{s}+\frac{2 x+5}{s^{2}}-\frac{2}{9 s^{5}}\right] \\
& v(x, y)=\frac{(8 x+2) y^{2}}{6}+\left(x^{2}+5 x\right)+\frac{(2 x+5) y}{2}-\frac{y^{4}}{108}
\end{aligned}
$$

Hence the general solution form is

$$
w=u+i v=-\frac{4 y^{3}}{9}+\left(x^{2}+5 x\right)-\frac{(2 x+5) y}{2}-\frac{y^{4}}{12}+i\left(\frac{(8 x+2) y^{2}}{6}+\left(x^{2}+5 x\right)+\frac{(2 x+5) y}{2}-\frac{y^{4}}{108}\right)
$$

Examples (1) and (2) are second order complex differential equations solved and equations (33) and (38) are the obtained solutions to the problem as stated.

\section{CONCLUSION}

Second order complex differential equation was considered in this paper, where the solution to such type of problems are not easily solved for. But with the derivation of the solution form of the complex differential equation using the Laplace Transform technique and then implementing it on the examples presented, the solution to the examples were easily arrived at as shown in equations (33) and (38) which is the general form of the solution giving the real and imaginary part of the complex differential equation.

\section{REFERENCES}

Dinesh V. (2018). Applications of Laplace Transformation for Solving Various Differential Equations with Variable Coefficients. International Journal for Innovative Research in Science \& Technology. 4(11), 2349-6010.

Gupta S, Kumar D \& Singh J. (2015). Numerical Study for Systems of Fractional Differential Equations Via Laplace Transform. Journal of the Egyptian Mathematical Society. 23, 256262.

Kazem S. (2013). Exact Solution of Some Linear Fractional Differential Equations by Laplace Transform. International Journal of Nonlinear Science. 16(1), 3-11.

Kexue L \& Jigen P. (2011). Laplace transform and fractional differential equations. Applied Mathematics Letters. 24(2011), 2019-2023. 
Mohamed M. A \& Torky M. S. (2013). Numerical solution of nonlinear system of partial differential equations by the Laplace decomposition method and the Pade approximation. American Journal of Computational Mathematics. 3(3), 175-184.

Murat D. (2017). On an Application of Laplace Transform. New Trends in Mathematical Sciences. 5(2), 193-198.

Saheed, A. (2014). Laplace transform method for solving differential equations.

Yin F. K, Han W. Y \& Song J. Q. (2013). Modified Laplace decomposition method for LaneEmden Type differential equations. International Journal of Applied Physics and Mathematics. 3(2), 98-102.

Yusufoglu E. (2006). Numerical Solution of duffing equation by the Laplace decomposition algorithm. Applied Mathematics and Computation. 177(2), 572-580. 